\title{
COMPOSIÇÃO QUÍMICA DAS SEMENTES DE Archontophoenix alexandrae H. Wendl. \& Drude (ARECACEAE) ${ }^{1}$
}

\author{
Maria Isabel Vallilo², Cybele de Souza Machado Crestana ${ }^{2}$, Sabria Aued-Pimentel $^{3}$, Mário Tavares $^{3}$, Edna $^{2}$ \\ Emy Kumagai ${ }^{3}$, Maria de Lima Garbelotti $^{3}$
}

\begin{abstract}
RESUMO - Sementes de Archontophoenix alexandrae extraídas de frutos coletados no Parque Estadual da Cantareira, São Paulo, foram analisadas quanto à composição química, ao perfil de ácidos graxos do óleo e aos teores de minerais, visando avaliar o seu potencial alimentício. Detectaram-se alto teor de fibras alimentares $(38,80 \%$ p/p) e umidade $(47,72 \%$ p/p). Na fração oleosa, apesar do baixo conteúdo de óleo encontrado $(2,74 \%$ p/p), predominaram os ácidos palmítico $(19,80 \% \mathrm{p} / \mathrm{p})$, entre os saturados, e oléico (42\% p/p) e linoléico $(13 \% \mathrm{p} / \mathrm{p})$, quanto aos insaturados. A presença, no óleo, de $\alpha$-tocoferol (vitamina E) equivalente a 4,0 mg 100

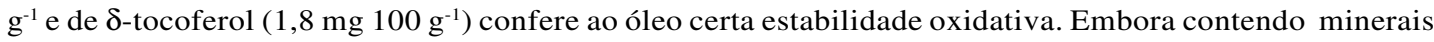
como K, P, S, Ca, Fe, Zn, Se e Cu, lipídios e fibras alimentares, a presença do elemento $\mathrm{Pb}\left(2,74 \mathrm{mg} \mathrm{kg}^{-1}\right)$ inviabiliza o consumo dessas sementes como alimento da avifauna e sinaliza contaminação antrópica no local de coleta.
\end{abstract}

Palavras-chave: palmeiras, composição centesimal, ácidos graxos, tocoferóis, elementos inorgânicos.

\section{SEED CHEMICAL COMPOSITION OF Archontophoenix alexandrae H. Wendl. \& Drude (ARECACEAE)}

\begin{abstract}
Seeds of Archontophoenix alexandrae, extracted from fruits collected at the Cantareira State Park, São Paulo, Brazil, were analyzed for chemical composition, oil fatty acids profiles and mineral content aiming to evaluate their nutritional potential. High values of dietary fibers $(38.80 \% \mathrm{w} / \mathrm{w})$ and humidity $(47.72 \%$ $w / w)$ were found. Palmitic acid $(19.80 \% \mathrm{w} / \mathrm{w})$ was predominant among the saturated oil fraction, and oleic $(42 \% \mathrm{w} / \mathrm{w})$ and linoleic $(13 \% \mathrm{w} / \mathrm{w}$ ) among the insaturated oil fraction. The presence of $\alpha$-tocopherol (vitamin

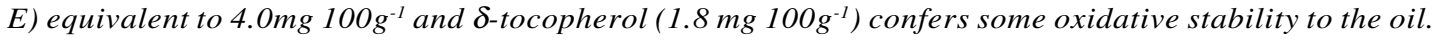
Even containing minerals such as $K, P, S, C a, Z n$, Se and $\mathrm{Cu}$, lipids and dietary fibers, the presence of $\mathrm{Pb}$ $\left(2.74 \mathrm{mg} \mathrm{kg}^{-1}\right)$ makes its consume unviable for avifauna, as well as it indicates anthropic contamination at the collect spot.
\end{abstract}

Key words: palms, centesimal composition, fatty acids, tocopherols and inorganics elements.

\section{INTRODUÇÃO}

Originária da Austrália, a palmeira Archontophoenix alexandre H. Wendl. \& Drude, da família Arecaceae, foi introduzida no Brasil como planta ornamental, onde hoje é mais comumente encontrada nas Regiões Sul e Sudeste, apresentando-se como uma espécie alternativa à produção de palmito, seja pelo esgotamento das reservas naturais de Euterpe edulis Mart., da Floresta Atlântica, ou pela contínua extração de Euterpe oleracea Mart., da Floresta Amazônica. É conhecida no Brasil sob os nomes de "seafortia", "palmeira-da-rainha", "palmeira-real-da-austrália-de-alexandre. Possui es-

\footnotetext{
${ }^{1}$ Recebido para publicação em 01.9.2003 e aceito para publicação em 10.8.2004.

${ }^{2}$ Instituto Florestal, Divisão de Dasonomia, Caixa Postal 1322, CEP 01059-970 São Paulo, SP. E-mail: <vallilo@uol.com.br>; <cybelemcrestana@ig.com.br>.

${ }^{3}$ Instituto Adolfo Lutz, Caixa Postal 1873, CEP 01059-970 São Paulo, SP. E-mail: <sabria_aued@yahoo.com>.
} 
tipe único, proeminente na base, elegante, rígido, anelado, com palmito grande, espesso e vistoso no topo; atinge entre 15 e $20 \mathrm{~m}$ de altura e $17 \mathrm{~cm}$ de DAP; as folhas são pinadas, grandes, recurvadas ou direitas, com folíolos longos, acinzentados na face inferior (LORENZI, 1996; RAMOS e HECK, 2001).

A inflorescência, envolta em espata, é do tipo panícula, com grande número de ráquilas pendentes; pistilo com três carpelos, ocorrendo um óvulo por carpelo; as flores têm perigônio verdadeiro e, quando novas, apresentam-se branco-creme. O florescimento dá-se no período primavera-verão. O fruto é do tipo drupa, globoso, unilocular, com mesocarpo carnoso, apresentando cor vermelha intensa quando maduro. Formase no período primavera-outono (LORENZI, 1996; RAMOS e HECK, 2001). A semente é única, lateral, com hilo linear. Apresenta formato arredondado, podendo ser levemente ovalada; após a despolpa torna-se amarelorosada, mostrando as fibras, que permanecem aderentes (LORENZI, 1996; ALVES e DEMATTÊ, 1987).

O clima exigido para o cultivo da espécie pode ser subtropical ou tropical, quente e úmido; a temperatura média anual é favorável entre 20 e $22^{\circ} \mathrm{C}$, não tolerando a ocorrência de geadas. Para que a planta tenha bom desenvolvimento, o índice pluviométrico pode variar entre 1.500 e $1.970 \mathrm{~mm}$ anuais, com boa distribuição sazonal. A espécie adapta-se bem às áreas planas ou onduladas e a diferentes tipos de solos, desde os extremamente arenosos até aqueles com alto conteúdo de argila, bem estruturados, sem compactação; tolera pH baixo (RAMOS e HECK, 2001).

O estipe de algumas espécies de palmeiras é composto de material plástico, como fibras, proteínas e polissacarídeos (celulose), o que lhes confere a forma, e de material nutritivo, que preenche o interior das células como açúcares e amido. No interior dos estipes de Metroxylon laeve Mart. e Raphia ruffia Mart., encontra-se amido em grande quantidade, cujo teor varia com a época do ano, com a floração e com a frutificação da planta. $M$. laeve perde o amido armazenado no estipe à época da floração, ocasião em que esse material é translocado, visando à formação de frutos (ALVES e DEMATTÊ, 1987). Mauritia flexuosa Linn. f., o buriti, é um exemplo de palmeira que apresenta o parênquima medular rico em açúcares e os frutos

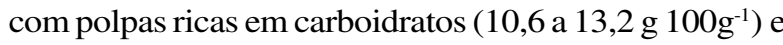
vitamina C (19,3 a 20,7 mg $\left.100 \mathrm{~g}^{-1}\right)$, conforme estudo de Tavares et al. (1996). Na polpa dessa espécie coletada em regiões próximas a Campo Grande, no Estado de Mato Grosso do Sul, Hiane et al. (1992) encontraram

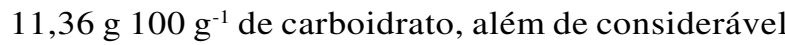

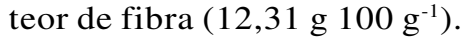

Com respeito à composição química dos frutos de A. alexandrae, particularmente do mesocarpo, pouco se conhece das palmeiras. No entanto, é comum observarse o interesse de diversas espécies de pássaros e roedores pelos frutos de A. alexandrae, entre eles a jandaia (Aratinga solstitialis), o papagaio (Amazona brasiliensis), o sanhaço (Thraupis $\mathrm{sp}$ ), a saíra (Microstur sp), o bem-te-vi (Pitangus sulphuratus) e o sabiá-poca (Turdus sp) (QUEIROZ, 1997; FRISCH, 2001; von IHERING, 2002), bem como do esquilo (Sciurus aestuans) e do macaco-prego (Cebus apella). Atraídos pela cor vermelha, os pássaros ingerem os frutos inteiros, regurgitando-os despolpados, atuando, assim, como dispersores da espécie. No processo de sucessão ecológica, é fundamental conhecer-se as inter-relações entre plantas e animais, para estabelecer um sistema auto-sustentável (REIS et al., 1999).

A interação entre $A$. alexandrae e a avifauna local é evidente, indicando que os frutos dessa palmeira contribuem como recurso alimentar à fauna passeriforme, contemplando o processo de dispersão natural dessa espécie.

O objetivo dessa pesquisa foi avaliar a composição química e o potencial das sementes de A. alexandrae como alimento da avifauna local e como matéria-prima industrial.

\section{MATERIAL E MÉTODOS}

Frutos maduros foram coletados do chão, no Parque Estadual da Cantareira, localizado ao norte da cidade de São Paulo, a 23 24' de latitude sul (S) e 46³6' de longitude oeste (W), e altitude média de $776 \mathrm{~m}$ (VENTURA et al., 1965/66). As sementes foram retiradas manualmente dos frutos, com o auxílio de morsa e espátula e acondicionadas em frascos plásticos, guardados sob refrigeração à temperatura de $5{ }^{\circ} \mathrm{C} \pm 2{ }^{\circ} \mathrm{C}$. A seguir, foram descascadas, trituradas e homogeneizadas em multiprocessador doméstico. As análises foram realizadas nos Laboratórios de Fitoquímica do Instituto Florestal e da Seção de Óleos, Gorduras e Condimentos do Instituto Adolfo Lutz, de São Paulo. 
A composição centesimal (umidade, resíduo mineral fixo, lipídios e proteínas) foi efetuada segundo as "Normas Analíticas do Instituto Adolfo Lutz" (INSTITUTO ADOLFO LUTZ, 1985), sendo os carboidratos calculados por diferença. Foi empregado o fator de 6,25 para a conversão do nitrogênio em proteínas. $O$ valor calórico foi calculado utilizando-se os seguintes fatores: nove para lipídios e quatro para proteínas e carboidratos (BRASIL, 1998a).

A determinação das fibras alimentares seguiu o método enzimático-gravimétrico da "Association of Official Analytical Chemists" (AOAC), modificado por Lee et al. (1992).

Para a análise dos ésteres metílicos dos ácidos graxos, o óleo foi extraído a frio, segundo o método modificado de Stansby e Lemon (1937). A conversão dos ácidos graxos em ésteres metílicos foi realizada conforme o método IUPAC 2301 (1987).

Os ésteres metílicos foram analisados em cromatógrafo a gás, marca Shimadzu, modelo GC-17A, com detector de ionização de chama. Os compostos foram separados em coluna capilar de sílica fundida SP 2340 de $60 \mathrm{~m}$, com diâmetro interno de $0,25 \mathrm{~cm}$ e espessura do filme de $0,20 \mu \mathrm{m}$. Foram obedecidas as seguintes condições de operação: temperatura programada da coluna de $60{ }^{\circ} \mathrm{C}(2 \mathrm{~min})$, taxa de aquecimento $15^{\circ} \mathrm{C} /$ min até $135^{\circ} \mathrm{C}(1 \mathrm{~min})$, taxa de aquecimento $3{ }^{\circ} \mathrm{C} / \mathrm{min}$ até $215^{\circ} \mathrm{C}(10 \mathrm{~min})$, temperatura do injetor de $230^{\circ} \mathrm{C}$, temperatura do detetor de $240^{\circ} \mathrm{C}$, velocidade linear do gás de arraste (hidrogênio) de $20 \mathrm{~cm} / \mathrm{s}$ e razão de divisão da amostra de 1:50. Os ácidos graxos foram identificados pela comparação dos tempos de retenção dos padrões puros de metil-ésteres de ácidos graxos e das amostras. A quantificação foi feita por normalização de área.

Os tocoferóis foram separados e quantificados por cromatografia líquida de alta eficiência (CLAE), de acordo com o método da AOCS Ce 8-89, 1996.

A determinação dos elementos inorgânicos foi realizada no Laboratório de Espectrometria do Instituto de Química da Universidade de São Paulo, por meio da digestão ácida em sistema focalizado Spex 350 assistido por radiação de microondas, com três repetições analíticas, utilizando o seguinte protocolo analítico: $1,0 \mathrm{~g}$ de amostra foi pesado e transferido para o copo de digestão, seguido da adição de 10 mL de $\mathrm{HNO}_{3}(65 \%)$ e $1 \mathrm{~mL}$ de $\mathrm{H}_{2} \mathrm{O}_{2}$ a $30 \%$ v/v. Em seguida, foram aplicadas potências de $45 \mathrm{~W}$ por $2 \mathrm{~min}, 60 \mathrm{~W}$ por $2 \mathrm{~min}$ e $75 \mathrm{~W}$ por $6 \mathrm{~min}$. Após o resfriamento, as amostras foram transferidas quantitativamente para balões volumétricos de $25 \mathrm{~mL}$ com água desionizada.

Os elementos $\mathrm{Na}, \mathrm{K}, \mathrm{Mg}, \mathrm{Ca}, \mathrm{P}, \mathrm{Al}, \mathrm{S}, \mathrm{Fe}, \mathrm{Ni}, \mathrm{Cu}$, As, $\mathrm{Zn}, \mathrm{Ba}, \mathrm{Mn}, \mathrm{Pb}, \mathrm{V}$ e $\mathrm{Se}$ foram identificados e quantificados nas amostras solubilizadas pela técnica da espectrometria de emissão ótica acoplada ao plasma indutivamente (ICP-OES), operando na potência de $1.200 \mathrm{~W}$, velocidade do gás de argônio refrigerante e auxiliar de 12 L.min ${ }^{-1}$ e 1,2 L.min ${ }^{-1}$, respectivamente; pressão no nebulizador de 26 psi; volume de introdução de amostra de 1,5 mL. $\mathrm{min}^{-1}$; e altura de observação de $12 \mathrm{~mm}$. A leitura dos elementos foi feita nos seguintes comprimentos de ondas $(\lambda \mathrm{s})$ em $\mathrm{nm}$ : $\lambda_{\mathrm{K}}=776,460 ; \lambda_{\mathrm{Mg}}=280,270 ; \lambda_{\mathrm{Pb}}=220,353 ; \lambda_{\mathrm{P}}=213,618$; $\lambda_{\mathrm{Al}}=396,152 ; \lambda_{\mathrm{Zn}}=213,855 ; \lambda_{\mathrm{S}}=180,73 ; \lambda_{\mathrm{Mn}}=257,610$; $\lambda_{\mathrm{Ba}}=233,527 ; \lambda_{\mathrm{Ni}}=221,647 ; \lambda_{\mathrm{Ca}}=422,673 ; \lambda_{\mathrm{Na}}=588,995$; $\lambda_{\mathrm{Fe}}=261,187 ; \lambda_{\mathrm{As}}=193,759 ; \lambda_{\mathrm{v}}=310,230 ; \mathrm{e} \lambda_{\mathrm{Se}}=203,985$, por meio de curvas analíticas elaboradas com soluções de trabalho multielementares preparadas nas concentrações de 0,$1 ; 0,5 ; 1,0 ; 5,0 ; 10,0 ; 50,0 ; 100,0$; e $500,0 \mu \mathrm{g} \mathrm{mL}^{-1}$ de cada elemento constituinte, em $\mathrm{HNO}_{3}$ a $1 \%$, por diluição da solução-estoque de concentração equivalente a $100 \mu \mathrm{g} \mathrm{mL}^{-1}$.

\section{RESULTADOS E DISCUSSÃO}

Verificaram-se altos teores de substâncias voláteis, fibras alimentares e de carboidratos na semente de A. alexandrae (Quadro 1). O valor calórico total

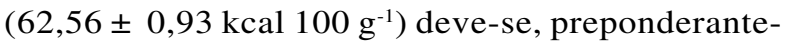
mente, aos carboidratos e lipídios, já que o teor de proteína pouco contribuiu nesse sentido. O teor de substâncias voláteis representa $47,72 \%$ da composição total da semente, indicando alto teor de água.

Comparando os resultados obtidos com os daqueles encontrados nas palmeiras Dypsis lutescens $\mathrm{H}$. Wendl., Phoenix roebelinii O’Brien., Phoenix canariensis Hort. Ex Chabaud e Syagrus romanzoffiana (Cham.) por Vallilo et al. (2001), observou-se que todas exibiram teores de umidade abaixo dos de A. alexandrae e, em consequiência, um valor calórico maior. Deve-se destacar o elevado conteúdo de fibras alimentares que, mesmo estando abaixo das de D. lutescens, P. roebelinii e $P$. canariensis, superou os valores recomendados pela Sociedade Brasileira de Alimentos e Nutrição (20 g/

R. Árvore, Viçosa-MG, v.28, n.5, p.676-679, 2004 
dia) e pela "American Dietetic Association"(30 g/dia), referenciados por Garbelotti (2000). As fibras alimentares desempenham importante papel fisiológico na redução das doenças intestinais, bem como na diminuição dos níveis de colesterol e de triglicérides séricos (TOGASHI e SCARBIERI, 1994).

O resultado obtido de $\alpha$-tocoferol (vitamina E) no óleo de $A$. alexandrae enquadra-se nos limites mínimos referenciados na literatura, nos óleos de soja (0,9-35,2\% mg $\left.100 \mathrm{~g}^{-1}\right)$ e de milho (2,3-57,3\% mg 100 $\left.\mathrm{g}^{-1}\right)$ e $\delta$-tocoferol, nos óleos de gergelim $(0,4-2,1 \% \mathrm{mg}$ $\left.100 \mathrm{~g}^{-1}\right)$ e de algodão (0,0-2,0\% mg $\left.100 \mathrm{~g}^{-1}\right)$ (FIRESTONE, 1999).

Apesar do pouco conteúdo de óleo encontrado $(2,74 \% \mathrm{p} / \mathrm{p})$, ressaltou-se a maior quantidade de ácidos graxos insaturados (A.G.I) $(56,01 \% \mathrm{p} / \mathrm{p})$ do que saturados $(43,03 \% \mathrm{p} / \mathrm{p})$, o que favoreceria o seu emprego para fins alimentícios, caso não se constatasse o pequeno rendimento encontrado (Quadro 2). Essa elevada insaturação do óleo confere maior instabilidade oxidativa nas condições normais de temperatura e luminosidade, no entanto a presença de $\alpha$-tocoferol (vitamina E), antioxidante natural, pode contribuir para a sua estabilidade química (FERRARI, 2001; WANG et al., 1998).

Entre os ácidos insaturados predominam os ácidos oléico e o linoléico, ressaltando-se que os valores obtidos no primeiro superam os encontrados nas quatro espécies

Quadro 1 - Composição centesimal e valor calórico de sementes de Archontophoenix alexandrae na amostra "in natura"

Table 1 - Centesimal composition and caloric value of Archontophoenix alexandrae in the sample "in natura"

\begin{tabular}{lc}
\hline Composição & Média \pm desvio-padrão \\
\hline$\left(\mathrm{g} 100 \mathrm{~g}^{-1}\right)$ & $(\mathrm{n}=3)$ \\
Substâncias voláteis a $105{ }^{\circ} \mathrm{C}$ & $47,72 \pm 0,05$ \\
Resíduo mineral fixo a $550{ }^{\circ} \mathrm{C}$ & $1,26 \pm 0,03$ \\
Lipídios & $2,74 \pm 0,04$ \\
Proteínas $(\mathrm{N}$ x 6,25$)$ & $3,17 \pm 0,07$ \\
Fibras alimentares & $38,80 \pm 0,21$ \\
Carboidratos totais* & $6,31 \pm 0,09$ \\
kcal $100 \mathrm{~g}^{-1}$ & \\
Valor calórico & $62,56 \pm 0,93$ \\
\hline
\end{tabular}

* - Calculado por diferença. já mencionadas, porém inferiores aos referidos para Mauritia flexuosa Linn. f. (buriti) (TAVARES et al., 1996). O ácido linoléico é importante do ponto de vista nutricional, visto que é considerado ácido graxo essencial, portanto não produzido pelo metabolismo animal, devendo ser administrado através da alimentação (TURATTI, 2000). Os teores encontrados nos dois ácidos enquadram-se na faixa de valores adotados tanto pelo regulamento técnico para fixação de identidade e qualidade de óleos e gorduras vegetais quanto pela comissão do Codex Alimentarius no óleo de amendoim (35,0-72,0\% p/p; 13,0-45,0\% p/p) (BRASIL, 1999; CODEX ALIMENTARIUS, 1993).

Em relação aos elementos inorgânicos, constatou-se a presença de cinco dos macrominerais $(\mathrm{K}, \mathrm{Ca}$, $\mathrm{Mg}, \mathrm{S}$ e P) dos oito considerados essenciais para o metabolismo dos organismos vivos (DEANGELIS, 1997), (Quadro 3). Entre eles, o K exibiu maior conteúdo, seguido de P, Ca, S e Mg.

Quadro 2 - Composição em ácidos graxos e de tocoferóis no óleo das sementes de Archontophoenix alexandrae Table 2 - Composition of fatty acids and tocopherols from Archontophoenix alexandrae seed's oil

\begin{tabular}{lc}
\hline Ácidos graxos & $\begin{array}{c}\text { Média } \pm \text { desvio-padrão } \\
(\% \text { p/p de ésteres metílicos) }\end{array}$ \\
\hline C8:0 (caprílico) & $0,14 \pm 0,01$ \\
C10:0 (cáprico) & $0,17 \pm 0,01$ \\
C12:0 (láurico) & $12,9 \pm 0,7$ \\
C14:0 (mirístico) & $6,7 \pm 0,5$ \\
C16:0 (palmítico) & $19,8 \pm 0,8$ \\
C16:1 (palmitoléico) & $0,25 \pm 0,01$ \\
C17:0 (margaroléico) & $0,18 \pm 0,01$ \\
C18:0 (esteárico) & $2,51 \pm 0,01$ \\
C18:1 (oléico) & $42 \pm 2$ \\
C18:2 (linoléico) & $13 \pm 2$ \\
C18:3 (linolênico) & $0,48 \pm 0,05$ \\
C20:0 (araquídico) & $0,22 \pm 0,01$ \\
C20:1 (eicosenóico) & $0,28 \pm 0,02$ \\
C22:0 (behênico) & $0,12 \pm 0,01$ \\
C24:0 (lignocérico) & $0,29 \pm 0,04$ \\
Outros & $0,37 \pm 0,01$ \\
Ácidos graxos saturados & 43,03 \\
Ácidos graxos insaturados & 56,01 \\
Tocoferois & $\left(\mathrm{mg} 100 \mathrm{~g}^{-1}\right.$ ) \\
$\alpha-$ Tocoferol & $4,0 \pm 0,4$ \\
$\delta$-Tocoferol & $1,8 \pm 0,4$ \\
\hline
\end{tabular}


Quadro 3-Concentrações médias dos elementos inorgânicos nas sementes de Archontophoenix alexandrae na amostra "in natura"

Table 3 -Means values of inorganics elements in the seeds of the Archontophoenix alexandrae of the sample "in natura"

\begin{tabular}{lc}
\hline Elemento & Média \pm desvio-padrão \\
\hline $\mathrm{Na}$ & $\left(\mathrm{mg} \mathrm{kg}^{-1}\right)$ \\
$\mathrm{Mg}$ & $58,17 \pm 0,21$ \\
$\mathrm{Al}$ & $219,33 \pm 1,15$ \\
$\mathrm{P}$ & $19,00 \pm 1,66$ \\
$\mathrm{~S}$ & $519,33 \pm 16,30$ \\
$\mathrm{Ca}$ & $306,66 \pm 23,18$ \\
$\mathrm{~V}$ & $452,66 \pm 6,66$ \\
$\mathrm{Mn}$ & $1,08 \pm 0,04$ \\
$\mathrm{Fe}$ & $5,61 \pm 0,11$ \\
$\mathrm{Ni}$ & $9,53 \pm 0,24$ \\
$\mathrm{Cu}$ & $0,385 \pm 0,041$ \\
$\mathrm{Zn}$ & $3,03 \pm 0,09$ \\
$\mathrm{As}$ & $4,52 \pm 0,41$ \\
$\mathrm{Se}$ & $0,996 \pm 0,021$ \\
$\mathrm{~Pb}$ & $1,50 \pm 0,14$ \\
$\mathrm{~K}$ & $2,44 \pm 0,23$ \\
$\mathrm{Ba}$ & $1058,33 \pm 9,71$ \\
& $24,00 \pm 0,62$ \\
\hline
\end{tabular}

Quanto aos micronutrientes essenciais, destacamse as presenças de $\mathrm{Cu}, \mathrm{Zn}$ e Se, que, segundo De Angelis (1997)e Fennema (1993), desempenham papéis importantes tanto na fisiologia do vegetal quanto do animal. Além disso, o $\mathrm{Cu}$ atua mesmo em quantidades pequenas, como catalisador de reações oxidativas na fração oleosa da semente (KARLESKIND apud FERRARI, 2001). O nível de $\mathrm{Cu}$ obtido no presente trabalho superou os encontrados nas palmeiras $D$. lutescens, $P$. roebelinii, $P$. canariensis e $S$. romanzoffiana e está abaixo do limite máximo permitido pela legislação brasileira de contaminantes químicos em alimentos de $10 \mathrm{mg} \mathrm{kg}$ ${ }^{1}$ (BRASIL, 1998b). Esse elemento é essencial ao metabolismo humano, mas, em concentrações elevadas, está associado a distúrbios do sistema nervoso central (LELIS et al., 2000).

O Se, também considerado micronutriente essencial, quando está associado à vitamina $\mathrm{E}$ ( $\alpha$-tocoferol) potencializa os processos antioxidantes da célula, evitando a formação de radicais livres considerados precursores do envelhecimento celular no homem. Sua deficiência na alimentação pode causar doenças como necrose do fígado e cegueira noturna (SHIMMA, 1995; ALCÂNTARA e WAGNER, 2000).
A presença, no entanto, do elemento $\mathrm{Pb}$, considerado bastante tóxico, está acima dos limites permitidos em alguns alimentos $\left(1,0 \mathrm{mg} \mathrm{kg}^{-1}\right.$ a $\left.0,3 \mathrm{mg} \mathrm{kg}^{-1}\right) \mathrm{se}-$ gundo a legislação brasileira relativa a contaminantes químicos (BRASIL, 1998b), o que inviabilizaria a utilização desses frutos ou sementes como alimento para a avifauna local e sinaliza problemas de contaminação ambiental na área de coleta.

\section{CONCLUSÕES}

As sementes da espécie Antophoenix alexandrae revelaram-se boa fonte de fibras alimentares e alto teor de água.

$\mathrm{Na}$ fração lipídica foram identificados cadeias carbônicas contendo de 8 a 24 carbonos, apresentando de zero a três insaturações. Os ácidos graxos insaturados representam $56,01 \%$ do total, sendo os mais abundantes os ácidos oléicos e linoleícos, assemelhando-se aos teores do óleo de amendoim. Os saturados representam $43,03 \%$ de $\mathrm{p} / \mathrm{p}$ do total, destacando-se os ácidos palmítico e láurico. Foram identificados e quantificados no óleo, $\alpha$-tocoferol e $\delta$ tocoferol.

Os valores razoáveis encontrados no $\mathrm{K}, \mathrm{P}, \mathrm{S}, \mathrm{Mg}$, $\mathrm{Ca}, \mathrm{Fe}, \mathrm{Zn}, \mathrm{Se}$ e $\mathrm{Cu}$ contribuem para a importância nutricional das sementes; no entanto, a presença do elemento $\mathrm{Pb}$ inviabiliza os frutos como alimento para a avifauna local e sinaliza contaminação antrópica na área de coleta.

\section{AGRADECIMENTO}

À Prof ${ }^{a}$ Dra. Elisabeth de Oliveira, do Instituto de Química da USP, pela colaboração nas análises químicas.

\section{REFERÊNCIAS BIBLIOGRÁFICAS}

ALCANTARA, S.; WAGNER, K. Introdução controlada de Se nas células da microalga Spirulina plateusis - cinética de absorção. In: ENCONTRO NACIONAL SOBRE CONTAMINANTES INORGÂNICOS, 7., SIMPÓSIO SOBRE ESSENCIALIDADE DE ELEMENTOS NA NUTRIÇÃO HUMANA, 2., 2000, Campinas. Livros de resumos. Campinas: ITAL, 2000. p.79-80.

R. Árvore, Viçosa-MG, v.28, n.5, p.676-679, 2004 
ALVES, M. R. P.; DEMATTÊ, M. E. S. P. Palmeiras-características botânicas e evolução. Campinas: Fundação Cargill, 1987.129p.

\section{AMERICAN OIL CHEMISTS SOCIETY - AOCS.} Official methods and reccomended practices of the american oil chemists' society. 4. ed. Champaign: 1996 (sections C, p. 15, I, p.37).

BRASIL, Leis, decretos, etc.- Portaria No 33/98 da Secretaria de Vigilância Sanitária do Ministério da Saúde. Diário Oficial da República Federativa do Brasil. Brasília, 30 mar. 1998. Seç. 1, n. 60-E, p.5-6. Adota os valores constantes das tabelas do anexo desta portaria como níveis de IDR-Ingestão Diária Recomendado para as vitaminas, minerais e proteínas.1998a

Portaria ${ }^{\circ} 685$ da Agência Nacional de Vigilância Sanitária (ANVISA) de 27 de agosto de 1998. Princípios gerais para o estabelecimento de níveis máximos de contaminantes químicos em alimentos. Diário Oficial da República Federativa do Brasil de 24 set.1998b. Disponível em: http://www.anvisa.gov.br/legis/ portaria685 98.htm Acesso em :29 mai. 2001.

-_-_- Resolução $n^{\circ}$ 482/99 da Diretoria Colegiada da Agência Nacional de Vigilância Sanitária. Diário Oficial, Brasília, 13 out. 1999. Seção 1, n.196 - E. p.82-87. Aprova o regulamento técnico referente a óleos e gorduras vegetais, constante de anexo desta resolução. (Anexo 2: Óleo de amendoim).

CODEX ALIMENTARIUS COMMISSION. Fats, oils and related products. 2. ed. Rome: FAO/WHO, 1993. v.8, p.13, 25, 57.

FENNEMA, R. C. Química de los alimentos. Zaragosa: Acribia, 1993.1095p.

FERRARI, R.A. Componentes minoritários de óleos vegetais. Óleos \& Grãos, n.58, p.20-28, 2001.

DE ANGELIS, R.C. Fisiologia da nutrição: fundamentos para nutrição e para desnutrição. EDART, v.2, n.1, p.55-76, 1977.
FIREstone, D. Physical and characteristics of oils fats and waxes. Champaign: AOCS Press, 1999. p.56-101.

FRISCH, J.D. Os doze cantos do Brasil-O folclore de 12 das mais belas aves brasileiras. São Paulo: Dalgas Ecoltec-Ecologia Técnica, 2001.64 p.

GARBELOTTI, M. L. Fibra alimentar e valor nutritivo de preparações servidas em restaurantes "por quilo" (Cerqueira César) de São Paulo, SP. 2000. 90f. Dissertação (Mestrado em Nutrição), Faculdade de Saúde Pública da Universidade de São Paulo, São Paulo, 2000.

HIANE, P. A. et al. Composição centesimal e perfil de ácidos graxos de alguns frutos nativos do estado do Mato Grosso do Sul. Boletim CEPPA, v. 10, n.1, p.35-42, 992.

INSTITUTO ADOLFO LUTZ - IAL. Normas Analíticas do Instituto Adolfo Lutz. Métodos químicos e físicos para análise de alimentos. 3. ed. São Paulo: IMESP, 1985. v.1, p.21-24, 266.

INTERNATIONAL UNION OF PURE AND APLLIED CHEMISTRY (IUPAC). Standard methods for analysis of oils, fats and derivatives. Blackwell Scientific publications. 1987; IUPAC Method 2.301; Report of IUPAC Working Group WG 2/87.

LEE, S. C.; PROSKY, L.; DEVRIES, J.W. Determination of total, soluble and insoluble dietary fiber in foods. Enzymatic-gravimetric method, Mês-TRIS Buffer: collaborative study. Jornal of Association of Official Analitical Chemistry International, v.75, p.395-416, 1992.

LELIS, K.L.A. et al. Determinação direta de Cu e $\mathrm{Cr}$ em urina por Espectrometria de Absorção Atômica em Forno de Grafite empregando Rutênio como Modificador Químico Permanente. In:

ENCONTRO NACIONAL SOBRE CONTAMINANTES INORGÂNICOS, 7.; SIMPÓSIO SOBRE ESSENCIALIDADE DE ELEMENTOS NA NUTRIÇÃO HUMANA, 2., 2000, Campinas. Livro de resumos. Campinas: ITAL, 2000. p.115-117. 
LOREnZI, H. Palmeiras no Brasil: exóticas e nativas. Nova Odessa: Plantarum, 1996. p.156.

QUEIROZ, L. R. S. 100 Animais Brasileiros publicados no Estadão. São Paulo: $O$ Estado de São Paulo, 1997.112 p.

RAMOS, M. G.; HECK, T.C. Cultivo da palmeira real-da-australia para a produção de palmito. Florianópolis: 2001. 32 p. (Boletim didático, 40).

REIS, A.; ZAMBONIN. R.M.; NAKAZONO, E.M. Recuperação de áreas florestais degradadas utilizando a sucessão e as interações plantaanimal. Reserva da Biosfera, n.14, p.42. 1999.

SHIMMA, E. Nutrição - nosso corpo mineral. Globo Ciência, n. 52, p.32-38, 1995.

STANSBY, M.E.; LEMON, J.M. Quantitative determination of oil in fish flesh. Indian Engineering Chemistry, v.9, n.7, p.341343, 1937.

TAVARES, M. et al. Composição química e estudo histológico do fruto de Buriti Nativo do Município de Buritizal, Estado de São Paulo.In: CONGRESSO BRASILEIRO DE CIÊNCIA E TECNOLOGIA DE ALIMENTOS, Poços de Caldas. Livro de resumos. Poços de Caldas: 1996. p.267-268.
TOGASHI, M.; SCARBIERI, V. C. Caracterização química parcial do fruto do Baru (Dipteyx alata Vog.) Ciência Tecnologia Alimentação, v.14, n.1, p.85-95, 1994.

TURATTI, J.M. Óleos vegetais como fonte de alimentos funcionais. Óleos \& Grãos, n.56, p.20-27, 2000.

VALLILO, M. I.et al. Composição química e o perfil de ácidos graxos das sementes de quatro espécies de palmeiras cultivadas no estado de São Paulo. Revista do Instituto Adolfo Lutz, v.13, n.2, p.147-154, 2001.

VENTURA, A.; BERENGUT, G.; VICTOR, M. A. M. Características edafo-climáticas das dependências do Serviço Florestal do Estado de São Paulo. Silvicultura em São Paulo, v. 4/5, n.4, p.57-140, 1965/1966.

VON IHERING, R. Dicionário dos animais do Brasil-Revisão Técnica de Denise Wilches Monsores-Difel- Rio de Janeiro: 2002. 558 p.

WANG, C. et al. Effects of steeping conditions during wet-milling on retention of tocopherols and tocotrienols in corn. Journal of American Oil Chemistry Society, v.75, p.609-613, 1998. 